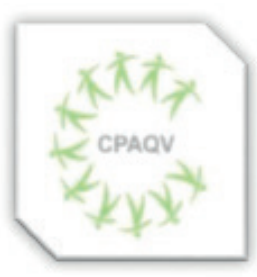

ISSN: 2178-7514
ARTIGO DE REVISÃO

\section{FISIOTERAPIA NO TRATAMENTO DE INCONTINÊNCIA URINÁRIA EM HOMENS PÓS- PROSTATECTOMIA: UMA REVISÃO INTEGRATIVA}

Physiotherapy in the treatment of urinary incontinence in men post - prostatectomy: an integrative review

Tereza Cristina dos Reis Ferreira ${ }^{2}$, Ana Eveni Rodrigues Aguiar ${ }^{1}$, Antônio Gabriel Pantoja Silva Santos ${ }^{1}$, Paula Thayna Soares Lima ${ }^{1}$, Pedro Victor Barriga Leopoldino ${ }^{1}$, Camila Alcântara Fernandes ${ }^{1}$,

Anne Beatriz Duarte da Conceição ${ }^{1}$, Bárbara Cristina Sousa da Silva ${ }^{1}$, Fernando Lucas Costa de Lima ${ }^{1}$, Tainah Lacerda Santos ${ }^{1}$

\title{
RESUMO
}

Objetivo: Identificar o papel da fisioterapia e as técnicas utilizadas para o tratamento de incontinência urinária (IU) em homens pós- prostatectomia. Métodos: Trata-se de um estudo de revisão integrativa da literatura. Esta revisão considerou estudos sobre o papel da fisioterapia no tratamento da IU em pacientes prostatectomizados, publicados entre janeiro de 2015 até outubro de 2020, em português e inglês. Os descritores em ciências da saúde (DeCS) utilizados para o idioma português são "Fisioterapia", "Incontinência urinária", "Prostatectomia", em inglês "Physical therapy specialty", "Urinary Incontinence", "Prostatectomy". As buscas foram realizadas nas bases eletrônicas PUBMED, SCIELO, LILACS E BIREME. Resultados: Foram encontrados 207 artigos, no entanto, para responder ao objetivo deste estudo foi selecionado 5 artigos. Os estudos analisados demonstram que a fisioterapia é benéfica para prevenir e tratar complicações decorrentes da prostatectomia, Além disso, a fisioterapia realiza fortalecimento da musculatura pélvica e reeducação muscular que reduz a IU. A fisioterapia utiliza eletroestimulação e cinesioterapia, para aumento da força muscular e redução das interferências geradas na vida dos paciente. Conclusão: Conclui-se a relevância da fisioterapia e seus recursos nos sintomas urinários, como: redução da perda urinária e do grau de incontinência, promovendo maior qualidade de vida aos pacientes.

Palavras-chave: Fisioterapia, Incontinência urinária, Prostatectomia

\begin{abstract}
Objective: To identify the role of physiotherapy and the techniques used to treat urinary incontinence (UTI) in men post-prostatectomy. Methods: This is an integrative literature review study. This review considered studies on the role of physiotherapy in the treatment of UI in prostatectomized patients, published between January 2015 and October 2020, in Portuguese and English. The descriptors in health sciences (DeCS) used for the Portuguese language are "Physiotherapy", "Urinary incontinence", "Prostatectomy", in English "Physical therapy specialty", "Urinary Incontinence", "Prostatectomy". The searches were carried out on the electronic bases PUBMED, SCIELO, LILACS AND BIREME. Results: 207 articles were found, however, to answer the objective of this study, 5 articles were selected. The analyzed studies demonstrate that physiotherapy is beneficial to prevent and treat complications resulting from prostatectomy. In addition, physiotherapy strengthens the pelvic muscles and muscle re-education that reduces UI. Physiotherapy uses electrostimulation and kinesiotherapy to increase muscle strength and reduce the interference generated in patients' lives. Conclusion: The relevance of physiotherapy and its resources in urinary symptoms is concluded, such as: reduction of urinary loss and the degree of incontinence, promoting a better quality of life for patients.
\end{abstract}

Keywords: Physical therapy specialty, Urinary Incontinence, Prostatectomy.

1- Discentes do Curso de Fisioterapia da Universidade do Estado do Pará (UEPA)

2- Fisioterapeuta, Dra em Ciências da Reabilitação (UNINOVE), Docente do Curso de Fisioterapia

da Universidade do Estado do Pará (UEPA).

Autor de correspondência

Tereza Cristina dos Reis Ferreira

tereza.reis@uepa.br

DOI: $\underline{10.36692 / v 12 n 3-29 r}$ 


\section{INTRODUÇÃO}

A próstata é um órgão exclusivo do sistema genital masculino, sendo aproximadamente do tamanho de uma bola de golfe e se encontra inferiormente a bexiga urinária circundando a uretra. Ela é responsável pela secreção de um líquido leitoso que envolve diversas substâncias com a função de nutrir e aumentar a viabilidade dos espermatozoides. $\mathrm{Na}$ glândula prostática, as morbidades mais comuns são a hiperplasia prostática benigna e o câncer de próstata. $^{1}$

Segundo o Instituto Nacional de Câncer José Alencar Gomes da Silva 2, o câncer de próstata é o segundo mais comum em homens, tendo como estimativa de novos casos cerca de 65.840 nos dados de 2020, sendo a sua maioria, no mundo todo, na faixa etária dos 65 anos ou mais. O estilo de vida, a idade e os fatores genéticos são as principais razões para o aumento de risco.

O tratamento para o CP se dá por meio do tempo de detecção do mesmo, em detecções precoces, onde o câncer ainda não atingiu outros órgãos (localizado), o tratamento é feito por cirurgia ou radioterapia, quando a doença se espalha (não localizado) é a partir da radioterapia ou da cirurgia combinada com terapia hormonal ${ }^{2}$.

A prostatectomia radical, é o procedimento mais utilizado em casos avançados de CP localizados que, geralmente, interfere muito na qualidade de vida do paciente. A incontinência urinaria pósprotatectomia é um dos principais impactos causados na saúde do homem, sendo na maioria das vezes, associada com disfunção erétil e hemorragia intraoperatoria. Esses fatores afetam diretamente na saúde mental do homem, uma vez que diminui a sua autoestima podendo causar depressão e ansiedade ${ }^{3}$.

A fisioterapia é fundamental na reabilitação de pacientes com IUPPR, já que tem resultados significantes no fortalecimento do assoalho pélvico masculino, aumentando o controle de sua micção. Estudos demonstram que por meio da realização da eletroterapia, terapia manual, cinesioterapia e exercícios domiciliares, combinados ou não, ajudam no fortalecimento muscular e no controle esfincteriano quando são realizados após no mínimo quatro meses da cirurgia ${ }^{4}$.

\section{MÉTODOS}

A pesquisa trata-se de uma revisão integrativa da literatura, dispondo da literatura científica existente sobre a fisioterapia no tratamento de incontinência urinaria em homens pós-prostatectomia como fonte de referências. Para a seleção das publicações foram utilizadas as bases de dados eletrônicas sendo estes: Scientific Eletronic Library online (SCIELO) e Literatura Latino-americana e do Caribe em Ciências da Saúde (LILACS), e US National Library of Medicine (PubMed). Os descritores utilizados para a busca dos dados foram: Fisioterapia; incontinência urinária; prostatectomia. Utilizando os termos contidos em meio aos critérios, de inclusão e exclusão. Foram aplicados como critérios de inclusão artigos de janeiro de 2010 até novembro de 2020, na língua portuguesa, espanhola e inglesa, com o tema relacionado a fisioterapia na incontinência urinária e prostatectomia, e a partir dos descritores citados, foram encontrados 207 artigos relacionados ao tema, e quando aplicados os critérios de exclusão: desconsiderou artigos que não proporcionavam o texto completo, sendo 25 selecionados buscando o objetivo principal do trabalho. (Quadro 1) 


\begin{tabular}{|c|c|}
\hline $\begin{array}{l}\text { Número de artigos encontrados com a } \\
\text { utilização dos descritores }\end{array}$ & 207 \\
\hline $\begin{array}{l}\text { Número de documentos adicionais } \\
\text { utilizando outras bases de dados }\end{array}$ & 0 \\
\hline $\begin{array}{l}\text { Número de artigos com aplicação dos } \\
\text { critérios de inclusão e exclusão }\end{array}$ & 25 \\
\hline $\begin{array}{l}\text { Numero de artigos com texto completo não } \\
\text { disponível }\end{array}$ & 13 \\
\hline $\begin{array}{l}\text { Numero de textos excluídos após leitura } \\
\text { completa }\end{array}$ & 7 \\
\hline $\begin{array}{l}\text { Numero de textos incluidos após leitura } \\
\text { completa }\end{array}$ & 6 \\
\hline
\end{tabular}

RESULTADOS E DISCUSSÃO trabalho abordam os principais achados dos seis

A partir da leitura completa dos estudos artigos científicos, de boa relevância, selecionados que estão expostos a seguir (Quadro 2). encontrados, os resultados presentes neste

\begin{tabular}{|c|c|c|}
\hline Autor/ ano & Título do trabalho & Resultados \\
\hline Straczynska et al. ${ }^{5}$ & $\begin{array}{l}\text { O impacto do treinamento dos músculos do assoalho } \\
\text { pélvico na incontinência urinária em homens após a } \\
\text { prostatectomia radical } \\
\text { sistemática }\end{array}$ & $\begin{array}{l}\text { os grupos experimentais receberam treinamento dos } \\
\text { músculos do assoalho pélvico(PFMT), enquanto os } \\
\text { grupos controle não receberam nenhuma intervenção } \\
\text { terapêutica. Em ambos os estudos, melhores } \\
\text { resultados foram observados no grupo experimental. } \\
\text { Cinco estudos compararam o PFMT guiado por } \\
\text { fisioterapeuta com o PFMT não supervisionado } \\
\text { realizado após receber instruções de treinamento } \\
\text { verbal. Em todos os estudos referenciados, melhores } \\
\text { resultados foram observados em pacientes } \\
\text { submetidos ao PFMT guiado por fisioterapeuta. Os } \\
\text { resultados do estudo demonstraram que um PFMT } \\
\text { guiado por fisioterapeuta reduziu significativamente a } \\
\text { duração e a gravidade da incontinência urinária }\end{array}$ \\
\hline De Lira et al. ${ }^{6}$ & $\begin{array}{l}\text { Efeitos do treinamento perioperatório dos músculos } \\
\text { do assoalho pélvico na recuperação precoce da } \\
\text { continência urinária e função erétil em homens } \\
\text { submetidos à prostatectomia radical: um ensaio } \\
\text { clínico randomizado }\end{array}$ & $\begin{array}{l}\text { Três meses após a prostatectomia radical(PR), a taxa } \\
\text { de incontinência urinária foi } 72,7 \% \text { no Grupo Controle } \\
\text { e } 70,0 \% \text { no Grupo Fisioterapia }(P>0,05) \text {. Não houve } \\
\text { diferença significativa nos escores do Questionário de } \\
\text { Consulta Internacional sobre Incontinência-Short Form } \\
\text { (ICIQ-SF) entre o Grupo Controle }(6,9 \pm 6,26) \text { e o } \\
\text { Grupo Fisioterapia }(7,0 \pm 5,12)(P=0,97) \text {. A atividade } \\
\text { eletromiográfica registrados em repouso e durante a } \\
\text { contração rápida do assoalho pélvico e a contração } \\
\text { sustentada do esfíncter anal externo antes e após a } \\
\text { PR foi semelhante nos dois grupos no início do estudo } \\
\text { e na avaliação de } 3 \text { meses pós-PR. Mas o grupo } \\
\text { Fisioterapia teve melhores pontuações. }\end{array}$ \\
\hline Zopf et al. ${ }^{7}$ & $\begin{array}{l}\text { Efeitos de um programa de exercícios } \\
\text { supervisionados de } 15 \text { meses sobre os resultados } \\
\text { fisicos e psicológicos em pacientes com câncer de } \\
\text { próstata após a prostatectomia: o estudo ProRehab }\end{array}$ & $\begin{array}{l}\text { As escalas funcionais do Questionário de Qualidade } \\
\text { de Vida do Câncer (EORTC QLQQ-C30) revelam } \\
\text { melhorias significativas no funcionamento físico, } \\
\text { papel, emocional e social ( } P<0,000, P<0,000, P= \\
0,020 \text { e } P=0,003 \text {, respectivamente) dentro do Grupo } \\
\text { de intervenção (GI). Apenas papel e funcionamento } \\
\text { social também melhoraram significativamente no } \\
\text { grupo controle (GC) ( } P=0,002 \text { e } P=0,015 \text {, } \\
\text { respectivamente). Em relação às escalas de sintomas, } \\
\text { a dispneia diminuiu significativamente no GI ( } P= \\
0,009 \text { ). Os resultados do Questionário de Qualidade } \\
\text { de Vida do Câncer de Próstata (EORTCQLQ-PR25) } \\
\text { mostraram uma diferença significativa do grupo ao } \\
\text { longo do tempo a favor do GI no escore de sintomas } \\
\text { urinários ( } P=0,027 \text { ). Melhorias significativas dentro } \\
\text { do grupo foram observadas nos sintomas urinários, } \\
\text { sintomas intestinais e função sexual ( } P=0,001, P= \\
0,007 \text { e } P=0,008 \text {, respectivamente) no GI desde o } \\
\text { início até o pós-teste. De acordo com os tamanhos de } \\
\text { efeito calculados dos resultados avaliados pelo } \\
\text { paciente, um efeito moderado a favor do IG pode ser } \\
\text { observado na escala de sintomas urinários. }\end{array}$ \\
\hline
\end{tabular}




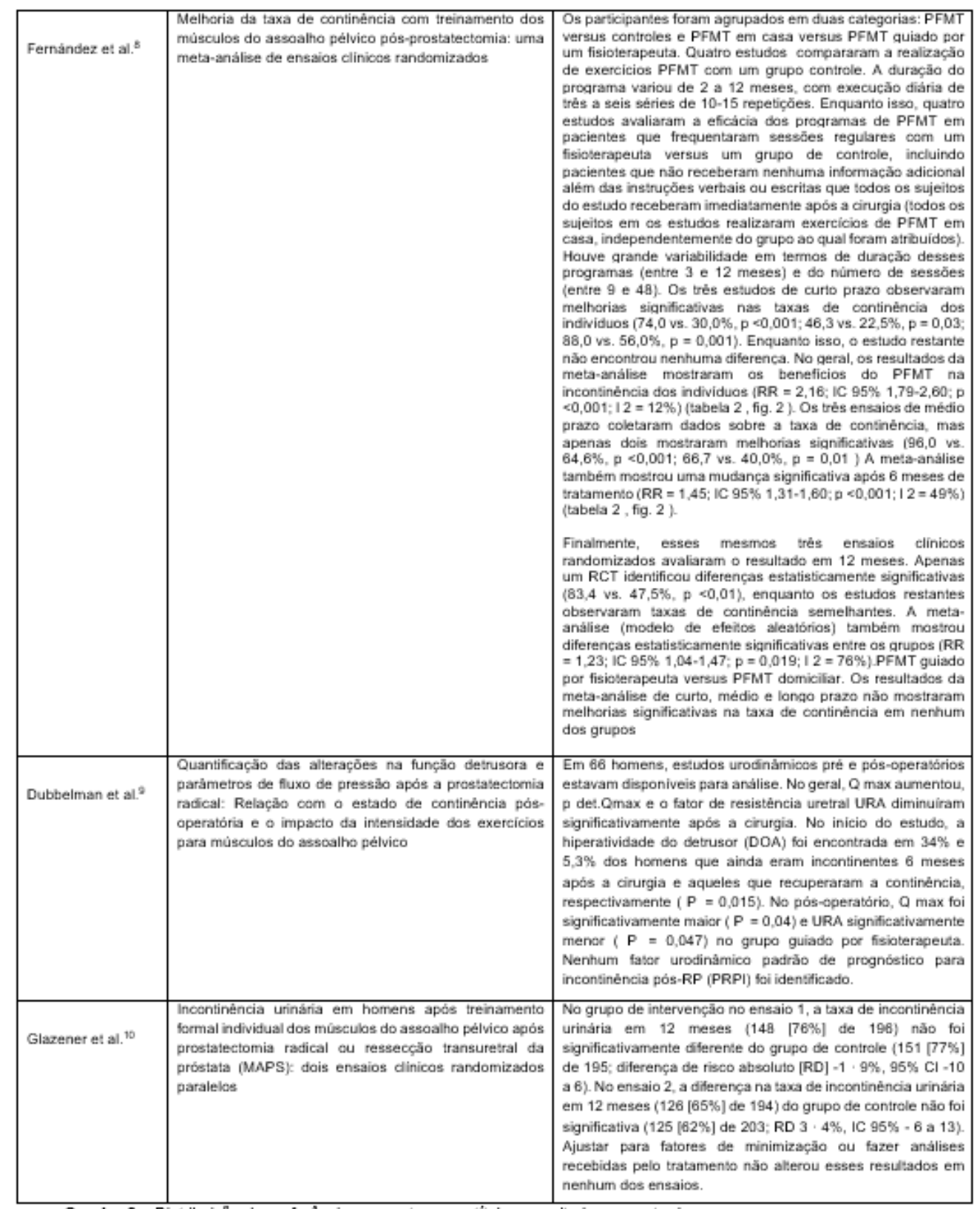

Quadro 2 - Distribuiçâo das referências por autor, ano, título e resultados encontrados.

Fonte: Pesquisa de campo, 2020.

O presente estudo analisou o papel da fisioterapia em pacientes com incontinência urinaria pós-prostectomia radical. Durante o levantamento de dados evidenciou-se a falta de estudos satisfatórios e completos que abordassem a intervenção fisioterapêutica nesse contexto da IU. Dessa maneira, os estudos selecionados e avaliados a partir dos nossos critérios de inclusão e exclusão demonstraram que o tratamento fisioterapêutico possui efeitos benéficos tanto em aspectos funcionais dos músculos do assoalho pélvico, que possui interferência direta sobre a incontinência urinaria, como também possui efeitos sobre aspectos psicológicos dos pacientes 
prostatectomizados.

Assim sendo, De Lira et al ${ }^{6}$ descreveu que o tratamento deve viabilizar os aspectos biopsicossociais dos pacientes. Entretanto, nos resultados desse estudo não foi considerada significativa a influência do acompanhamento fisioterapêutico na melhora do paciente, levando em consideração os resultados do grupo controle do estudo.

Em contrapartida, Straczynska et al5 analisou que o impacto do treinamento dos músculos do assoalho pélvico na incontinência urinária em homens após a prostatectomia radical foi mais efetivo com a intervenção fisioterapêutica, o que também foi comprovado por meio de escalas funcionais do Questionário de Qualidade de Vida do Câncer (EORTC QLQ-C30), confirmando que a atuação fisioterapêutica e influente também em aspectos psicológicos além do aspecto funcional. ${ }^{7}$

Portanto, de acordo com a literatura a atuação fisioterapêutica pode ter total influência sobre os aspectos musculares e psicológicos dos pacientes prostatectomizados, entretanto esse processo de reabilitação deve ser viabilizado a longo prazo, visto que Dubbelman et al 9, descreve que a alterações de músculos do assoalho pélvico ainda pode ser evidenciada após alguns meses após a cirurgia, como por exemplo a hiperatividade do detrusor (DOA) que foi identificada em 34\% dos pacientes, e também, 5,3\% dos homens ainda podem possuir incontinência urinaria .

\section{CONCLUSÃO}

A incontinência urinária é uma doença muito presente em nosso cotidiano, onde em alguns casos os pacientes se encontram com um sentimento de vergonha em relação a patologia e acabam gerando transtornos em sua vida, chegando até ao isolamento social, dificultando assim, que a mesma seja tratada de forma adequada. Dessa maneira, concluímos que a presente revisão integrativa permitiu demonstrar que a atuação da fisioterapia no tratamento da incontinência urinaria em homens pósprostatectomia apresenta melhora significativa em suas condições clínicas, tanto na reeducação das perdas urinarias quanto da qualidade de vida dos portadores, independente da terapêutica aplicada. Dessa forma, a fisioterapia porintermédio dos seus recursos, torna-se um instrumento indispensável para a elaboração de planos de tratamento para a reabilitação do paciente com incontinência urinária pós-prostatectomia. Contudo, novos estudos devem ser elaborados abordando esses temas, com o intuito de comprovar e divulgar essa terapia conservadora pouco difundida entre os demais profissionais da saúde.

\section{REFERÊNCIAS}

1- TORTORA, G.J.; DERRICKSON, B. Princípios de anatomia e fisiologia. 12a ed. Rio de Janeiro, Guanabara Koogan, 2010.

2- INCA, Instituto Nacional de Câncer. Câncer de próstata. 2020. Disponível em: https://www.inca.gov. br/tipos-de-cancer/cancer-de-prostata. Acesso em: 09 out. 2020.

3- BERNARDES, Mariana Ferreira Vaz Gontijo et 
al. Impacto da incontinência urinária na qualidade de vida de indivíduos submetidos à prostatectomia radical. Rev. Latino-Am. Enfermagem, Ribeirão Preto , v. 27, e3131, 2019

4-.SODRÉ, Danielle Santana Macêdo. RADIOFREQUENNCIA NO TRATAMENTO DA INCONTINÊNCIA URINÁRIA PÓSPROSTATECTOMIA RADICAL: ESTUDO PILOTO. 2017. 82 f. Dissertação (Mestrado) - Curso de Pós-Graduação em Medicina e Saúde Humana, Escola Bahiana de Medicina e Saúde Pública, Salvador, 2017.

5- STRACZYNSKA A, et al. The Impact Of Pelvic Floor Muscle Training On Urinary Incontinence In Men After Radical Prostatectomy (RP) - A Systematic Review. Clin Interv Aging, 2019; 14: 1997-2005

6-DE LIRA GHS et al. Effects of perioperative pelvic floor muscle training on early recovery of urinary continence and erectile function in men undergoing radical prostatectomy: a randomized clinical trial. Int Braz J Urol, 2019; 45(6): 1196-1203.

7- ZOPF EM et al. Effects of a 15-Month Supervised Exercise Program on Physical and Psychological Outcomes in Prostate Cancer Patients Following Prostatectomy: The ProRehab Study. Integr Cancer Ther, 2015; 14(5): 409-18.

8- FERNÁNDEZ, R.A. et al. Improvement of continence rate with pelvic floor muscle training postprostatectomy: a meta-analysis of randomized controlled trials. Urol Int. 2015; 94(2):125-32.

9 - DUBBELMAN, Y. et al. Quantification of changes in detrusor function and pressure-flow parameters after radical prostatectomy: relation to postoperative continence status and the impact of intensity of pelvic floor muscle exercises. Neurourol Urodyn. 2012; 31(5): 637-41.

10 - GLAZENER, C. et al. Urinary incontinence in men after formal one-to-one pelvic-floor muscle training following radical prostatectomy or transurethral resection of the prostate (MAPS): two parallel randomised controlled trials. Lancet. 2011; 378(9788): 328-37.

OBSERVAÇÃO: Os autores declaram não existir conflitos de interesse de qualquer natureza. 